\title{
PO2-3-7
}

Poster session

\section{In patients with diabetes mellitus (DM), but not in patients without DM, angiotensin II is a vasospasm inducer in both venous and arterial conduits under coronary artery bypass grafting}

Tatsuo Shiba ${ }^{1}$, Atsuko Yokota ${ }^{2,5}$, Shuji Gamoh ${ }^{1}$, Naoko Tanaka-Totoribe ${ }^{3}$, Masachika Kuwabara ${ }^{4}$, Eisaku Nakamura ${ }^{5}$, Takahiro Hayase ${ }^{2}$, Kunihide Nakamura ${ }^{5}$, Hiroaki Hisa ${ }^{6}$, Ryuichi Yamamoto ${ }^{1}$

${ }^{I}$ First Dept. Pharmacol., Sch. Pharmaceut., Kyushu University of Health and Welfare, Japan, ${ }^{2}$ Dept. Cardiovasc. Surg. Miyazaki Pref., Japan, ${ }^{3}$ Dept. Biopharmaceutics, Sch. Pharmaceut. Sci., Kyushu University of Health and Welfare, Japan, ${ }^{4}$ Kuwabara Clinic, Japan, ${ }^{5}$ Dept. Surg., Fact. Med., Miyazaki University, Japan, ${ }^{6}$ Second Dept. Pharmacol., Sch. Pharmaceut. Sci., Kyushu University of Health and Welfare, Japan

Coronary artery bypass grafting (CABG) remains the gold standard therapy for severe coronary artery disease. Diabetes mellitus $(\mathrm{DM})$ is an important risk factor for adverse outcomes of CABG, but the detailed mechanism is still unclear. Angiotensin II (AngII) is a part of the renin-angiotensin-aldosterone system and plays important roles in regulating the vascular tone and the pathogenesis of cardiovascular diseases. Thus, AngII may be closely related to the vasospasm. To elucidate whether AngII is a risk factor for vasospasm undergoing CABG, we evaluated the constrictive reactivity to AngII in isolated human endothelium-denuded saphenous vein (SV) and internal thoracic artery (ITA) harvested from patients with and without DM. Human SV and ITA tissues were obtained from patients undergoing CABG at Miyazaki Prefectural Nobeoka Hospital. The Ethics Committees of Kyushu University of Health and Welfare and Miyazaki Prefectural Nobeoka Hospital approved the project. AngII-induced constrictions of both vessels were significantly greater in the DM group than in the non-DM group. In non-DM group, AngII-induced constrictions of both vessels were very small, suggesting that AngII is not a risk factor for CABG in patients without DM. AngII acts primarily through the AngII type 1 (AT1) receptor to exert the most of its biological effects, including the constriction of vascular smooth muscle. The protein levels of AT1 receptor in the membrane fractions of the SV and ITA smooth muscle cells of patients with DM were significantly increased as compared with those of the patients without DM. These results suggest that the mechanism of hyper-reactivity to AngII in the SV and ITA from patients with DM is due to at least, the increase in the amount of AT1 receptor on membrane of the SV and ITA smooth muscle cells. Furthermore, these results indicate that AngII is a potent inducer of SV and ITA vasospasms in patients with DM, but not in patients without DM. Thus, the use of AngII receptor blocker (ARB) or angiotensin converting enzyme inhibitor (ACEI) in patients undergoing CABG, particularly among DM patients, undoubtedly contributes to the prevention of graft spasm, aside from their established such as antihypertensive and cardio-protective effects. 